Original Article

\title{
The effects of computer-assisted cognitive rehabilitation on Alzheimer's dementia patients memories
}

\author{
Jung-Ha Hwang, OT, PhD ${ }^{1)}$, Hyun-Gyu Cha, PT, PhD $\left.{ }^{2}\right)^{*}$, Young-Seok Cho, OT, $\mathrm{PhD}^{3)}$, \\ TAe-Sue Kim, PT, OT, PhD ${ }^{3)}$, Hyuk-Shin Cho, PT, $\mathrm{PhD}^{4)}$ \\ 1) Department of Occupational Therapy, Gwang Yang Health College, Republic of Korea \\ 2) Department of Physical Therapy, College of Kyungbuk: 77 Daehak-ro, Yeongju, Gyeongsangbuk-do \\ 750-712, Republic of Korea \\ 3) Department of Occupational Therapy, Gumi University, Republic of Korea \\ 4) Department of Physical Therapy, Wonkwang Health Science University, Republic of Korea
}

\begin{abstract}
Purpose] The purpose of the present study was to conduct Computer-Assisted Cognitive Rehabilitation (COMCOG) to examine the effects of COMCOG on Alzheimer's dementia patients' memories. [Subjects] Thirty-five patients diagnosed with Alzheimer's dementia received COMCOG for 30 minutes per day, five days per week for four weeks. [Methods] Before and after the COMCOG intervention, subjects' cognitive functions were evaluated using the Cognitive Assessment Reference Diagnosis System (CARDS) and Mini-Mental State Examination-Korea (MMSE-K) test. [Results] According to the results of the evaluation, among the CARDS scores of the subjects who received COMCOG, the scores of the delayed 10-word list, delayed 10-object list, recognition 10 -object, and recent memory significantly increased while the scores of recognition 10-word significantly decreased after intervention compared to before intervention. In addition, among the MMSE-K items, the orientation, registration, and recall showed significant increases. [Conclusion] Based on these results, delay in the progress of memory deterioration can be expected when COMCOG is conducted for Alzheimer's dementia patients who show declines in cognitive functions.

Key words: Alzheimer's, Computer-Assisted Cognitive Rehabilitation, Memory
\end{abstract}

(This article was submitted May 15, 2015, and was accepted Jun. 17, 2015)

\section{INTRODUCTION}

In the case of Alzheimer's dementia, cognitive disorders such as disorders in attention, start, plans, organization, problem solving ability, reasoning power, calculation ability, and memory appear before vascular dementia symptoms, such as motor or behavior disorders ${ }^{1}$. Alzheimer's dementia patients' characteristics are impairment of the explicit memory necessary to obtain and maintain new information and decline in memory which inhibits subjects ability to perform daily living activities ${ }^{2,3)}$.

Cognitive rehabilitation intervention methods for dementia patients generally implemented at clinics include Computer-Assisted Cognitive Rehabilitation (COMCOG), memory rehabilitation, cognitive stimulation, and psychological therapy ${ }^{2}$. Among them, COMCOG is currently used at clinics widely, and has the advantages of being easily

*Corresponding author. Hyun-Gyu Cha (E-mail: niceguychatty@hanmail.net)

C2015 The Society of Physical Therapy Science. Published by IPEC Inc. This is an open-access article distributed under the terms of the Creative Commons Attribution Non-Commercial No Derivatives (by-ncnd) License $<$ http://creativecommons.org/licenses/by-nc-nd/3.0/>. adjusted to individual patients' cognitive levels and accurate results and immediate feedback for judging the performance of patients ${ }^{2)}$.

In a study that conducted COMCOG for Alzheimer's dementia patients, delay in the progress of cognitive function disorders was identified ${ }^{4}$. In another study, it was reported that after conducting COMCOG for Alzheimer's dementia patients for four weeks, the number of errors in task performance decreased ${ }^{5}$. However, studies that have conducted COMCOG for Alzheimer's dementia patients and examined its memory improvement effects have been are in South Korea. The purpose of the present study was to conduct COMCOG for Alzheimer's dementia patients' to examine its effects on memory and to provide basic data for the effects of COMCOG on Alzheimer's dementia patients at clinics.

\section{SUBJECTS AND METHODS}

The present study was conducted with 35 in patients diagnosed with Alzheimer's dementia. Criteria for selection of subjects for the present study were: a Clinical Dementia Rating (CDR) in the range of 0.5-2, a Mini-Mental State Examination-Korea (MMSE-K) score of 23 points or lower, and no history of neurological disease. Participants who met the inclusion criteria and agreed to participate in the study 
Table 1. Pre- and post- intervention values of the CARDS and MMSE-K ( $=35)$

\begin{tabular}{lcc}
\hline & pre & post \\
\hline CARDS & & \\
Delayed 10-word list & $10.28 \pm 14.64^{\mathrm{a}}$ & $23.71 \pm 21.15^{* * *}$ \\
Delayed 10-object list & $2.00 \pm 5.31$ & $22.00 \pm 19.37^{* * *}$ \\
Recognition of 10 words & $34.00 \pm 33.97$ & $14.00 \pm 22.90^{* *}$ \\
Recognition of 10 objects & $34.57 \pm 19.30$ & $52.00 \pm 24.22^{* * *}$ \\
Recent memory & $36.85 \pm 28.59$ & $49.42 \pm 26.22^{* *}$ \\
MMSE-K & & \\
Orientation & $4.71 \pm 1.58$ & $5.48 \pm 2.68^{*}$ \\
Registration & $2.48 \pm 0.91$ & $2.68 \pm 0.79^{*}$ \\
Recall & $1.00 \pm 1.69$ & $1.62 \pm 2.01^{*}$ \\
Language & $6.97 \pm 1.52$ & $7.00 \pm 1.59$ \\
Attention and calculation & $1.45 \pm 1.55$ & $1.85 \pm 1.68$ \\
\hline${ }^{\mathrm{a}}$ Mean $\pm \mathrm{SD},{ }^{*} \mathrm{p}<0.05,{ }^{* *} \mathrm{p}<0.01{ }^{* * *} \mathrm{p}<0.001$, CARDS: Cognitive assessment refer-
\end{tabular}

ence diagnosis system; MMSE-K: Mini-mental state examination-Korea

received a general explanation of the procedures, and gave their written informed consent. All procedures were reviewed and approved by the Institutional Ethics Committee of Cheongju University.

The tester provided education before the intervention so that the subjects could learn to use computers and manipulate the mouse by themselves. The COMCOG program consists of items such as simple recognition memory, simple spatial memory, sequential recall memory, language recall memory, combined recall memory, language categorization memory, and language integration memory, and is intended to improve memories. This program was conducted for 30 minutes per day, five times per week for four weeks.

The Cognitive Assessment Reference Diagnosis System (CARDS) is a cognitive test tool that includes individual tests consisting of seven memory test items and five non-memory test items. The five memory test items consist of recognition of 10 words, delayed 10-word list, recent memory, delayed 10 -object list, and recognition of 10 objects. In the present study, to measure memories, memory-related tests of five items were conducted. The test-retest reliability and interrater reliability were very high at $0.74-0.99^{2}$ ).

MMSE-K is the Korean version of a standardized cognitive evaluation tool and is a dementia screening tool used to evaluate elderly persons' cognitive disorders and whether they have dementia. It consists of a total of 12 questions with a maximum possible score of 30 points: 24 points or higher indicates a normal state, 20-23 points indicates suspected dementia, and 19 points or lower indicates dementia. The inter-rater reliability of this test tool is very high at $0.99^{5}$ ).

In the present study, data were analyzed using SPSS 18.0 and the paired t-test was conducted to test average differences in memories between before and after intervention. Significance was accepted for values of $p<0.05$.

\section{RESULTS}

Thrity-five subjects participated in the present study, 14 males and 21 females. The subjects' ages were distributed as follows: $60-69$ years 5 (14.3\%), 70-79 years $18(51.4 \%)$,
$80-89$ years $11(31.4 \%), 90$ years or older $1(2.9 \%)$. The number of subjects with CDR class 0.5 was $7(20 \%)$, with class 1 was 17 (48.6\%), and with class 2 was 11 (31.4\%). The subjects' dementia duration was $20.94 \pm 9.66$ months and their average MMSE-K score was $16.63 \pm 4.35$ points.

After conducting COMCOG, among CARDS items, the scores of recognition of 10 -words decreased significantly $(p<0.01)$, while the scores of all the other variables increased significantly $(\mathrm{p}<0.01)$.

Among MMSE-K items, orientation, registration, and recall showed significant increases $(p<0.05)$ (Table 1$)$.

\section{DISCUSSION}

In the present study, after conducting COMCOG, Alzheimer's dementia patients' CARDS scores of the delayed 10 -word list, the delayed 10-object list, recognition of 10 objects, and recent memory items significantly increased. These results are consistent with the results of previous studies in which Alzheimer's dementia patients' memories were improved through $\mathrm{COMCOG}^{6}$.

However, in the present study, the scores of recognition of 10 words, that is understanding the meanings of words, significantly decreased after intervention unlike the other items and it is our assumption that the characteristics of Alzheimer's dementia are reflected in this result. These results are similar to the results of previous studies indicating that in the results of cognitive rehabilitation intervention, Alzheimer's dementia patients' scores in the recognition of 10 words item were shown to be lower than those of the general elderly persons or groups with other kinds of dementia, as Alzheimer's dementia patients' ability to distinguish between words and to accurately understand the meanings of words is impaired ${ }^{7)}$.

In the results of the present study, the scores of the delayed 10 -word list item were increased by the intervention while the scores of the recognition of 10 words decreased. A likely reason for this is the characteristics of the evaluation. Recognition of 10 words requires an understanding of the relevant words to re-recognize the words, whereas in the 
delayed 10-word list only recall of words that were heard even if the words may not be properly understood ${ }^{8}$.

Based on the results of previous studies of the characteristics of Alzheimer's dementia patients, it is thought that the effects of COMCOG on Alzheimer's dementia patients' recognition of 10 words ability is lower than those on other kinds of memories.

In a study conducted by Cipriani et al., it was reported that when COMCOG was conducted to 10 Alzheimer's dementia patients, their MMSE scores significantly increased ${ }^{6}$.

The results of the present study are similar and among the MMSE-K items, the memory item showed a significant increase. These results provide evidence that COMCOG is effective at improving memories of Alzheimer's dementia patients. Based on these results, when in the deterioration of memories can be expected COMCOG is conducted for Alzheimer's dementia patients showing declines in cognitive functions.

\section{REFERENCES}

1) Bickel H, Cooper B: Incidence and relative risk of dementia in an urban elderly population: findings of a prospective field study. Psychol Med, 1994, 24: 179-192. [Medline] [CrossRef]

2) Yu F, Rose KM, Burgener SC, et al.: Cognitive training for early-stage Alzheimer's disease and dementia. J Gerontol Nurs, 2009, 35: 23-29. [Medline] [CrossRef]

3) Cha HK, Ji SG, Kim MK, et al.: Effect of transcranial direct current stimulation of function in patients with stroke. J Phys Ther Sci, 2014, 26: 363 365. [Medline] [CrossRef]

4) Galante E, Venturini G, Fiaccadori C: Computer-based cognitive intervention for dementia: preliminary results of a randomized clinical trial. G Ital Med Lav Ergon, 2007, 29: B26-B32. [Medline]

5) Hofmann M, Rösler A, Schwarz W, et al.: Interactive computer-training as a therapeutic tool in Alzheimer's disease. Compr Psychiatry, 2003, 44: 213-219. [Medline] [CrossRef]

6) Cipriani G, Bianchetti A, Trabucchi M: Outcomes of a computer-based cognitive rehabilitation program on Alzheimer's disease patients compared with those on patients affected by mild cognitive impairment. Arch Gerontol Geriatr, 2006, 43: 327-335. [Medline] [CrossRef]

7) Simons JS, Graham KS, Hodges JR: Perceptual and semantic contributions to episodic memory: evidence from semantic dementia and Alzheimer's disease. J Mem Lang, 2002, 47: 197-213. [CrossRef]

8) Terry RD, Katzman R, Bick KL: Alzheimer's disease. New York: Raven Press, 1994. 